\title{
A Compact Low-loss Magic-T using Microstrip-Slotline Transitions
}

\author{
Kongpop U-yen ${ }^{1}$, Edward J. Wollack ${ }^{1}$, Samuel H. Moseley ${ }^{1}$, John Papapolymerou ${ }^{2}$ and Joy Laskar ${ }^{2}$ \\ ${ }^{1}$ NASA Goddard Space Flight Center, Greenbelt, Maryland, 20771, USA \\ ${ }^{2}$ Georgia Institute of Technology, Atlanta, Georgia, 30332, USA
}

\begin{abstract}
The design of a compact low-loss magic-T is proposed. The planar magic- $T$ incorporates the compact microstrip-slotline tee junction and small microstrip-slotline transition area to reduce slotline radiation. The experimental results show that the magic- $T$ produces broadband in-phase and out-of-phase power combiner/divider responses, has an average in-band insertion loss of $0.3 \mathrm{~dB}$ and small in-band phase and amplitude imbalance of less than $\pm 1.6^{\circ}$ and $\pm 0.3 \mathrm{~dB}$, respectively.

Index Terms - Microstrip circuits, passive circuits, power combiners, power dividers, slotline transitions.
\end{abstract}

\section{INTRODUCTION}

Planar magic-Ts are used in microwave integrated circuits to split or combine in-phase and out-of-phase signals. Applications include balanced-mixers, discriminators, interferometers, and beam-forming networks. Desirable properties of a magic- $T$ include: wide bandwidth phase and amplitude balance, low insertion loss, high isolation, compact size, and fabrication simplicity.

Several techniques have been developed to provide broadband response to a magic-T. Co-planar waveguide (CPW) or microstrip (MS) to slotline (SL) mode conversion techniques [1]-[4] are widely incorporated in a magic- $T$ to produce a broadband out-of-phase power combiner or divider such that the slotline transmission becomes the main part of these magic-Ts. Since a slotline has less field confinement than a microstrip or a CPW, slotline radiation can cause high insertion loss in these magic-Ts. In addition, the magic-T constructed from CPW transmission lines requires the bonding process for air bridges which increases fabrication complexity. Although aperture coupled magic-Ts [5] have a small slot area, they require three metal layers.

In this paper, we propose a magic- $\mathrm{T}$ design that incorporates a MS-SL tee junction and a MS-SL transition using minimum size SL terminations such that the magic- $T$ is compact and has less slotline radiation loss than the previously propose magicTs [1]-[4]. Finally, the hardware prototype magic-T operating at $10 \mathrm{GHz}$ is developed to evaluate the magic-T's performance.

\section{MAGIC-T CONFIGURATIONS AND POWER COMBINING/DIVIDING SCHEME}

The proposed magic-T, shown in Fig. 1, consists of the quarter-wavelength $(\lambda / 4)$ microstrip lines with the characteristic impedances of $Z_{1}, Z_{2}$ and $Z_{\imath}$. The $Z_{1}$ line with the

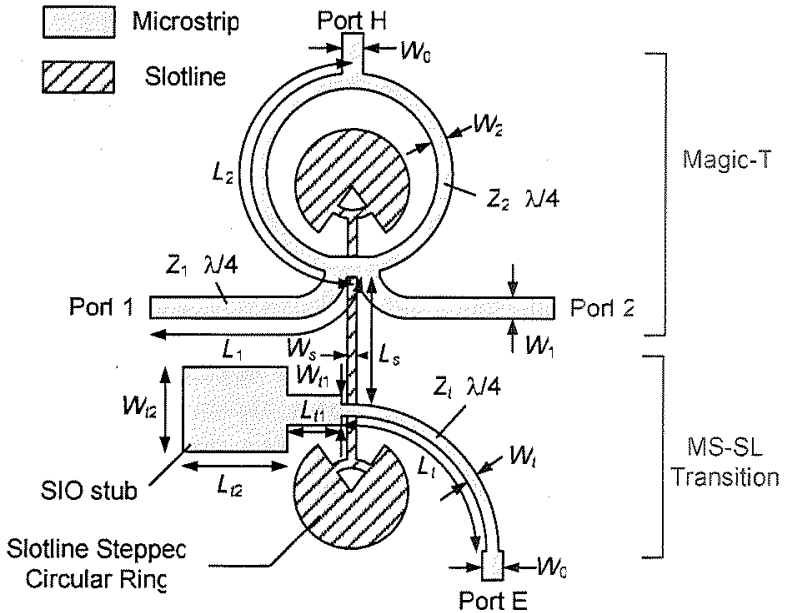

Fig. 1. The proposed $10 \mathrm{GHz}$ magic-T with microstrip ports on the $0.25 \mathrm{~mm}$-thick Duroid 6010 substrate. Dimensions: $5 \mathrm{~mm} \times 6.2 \mathrm{~mm}$.

length of $L_{1}$ is used to transform the characteristic impedance $Z_{0}$ at port 1 or port 2 to a slotline impedance $\left(Z_{s}\right)$ at the center of the structure. $Z_{2}$ and $Z_{t}$ lines (with the length of $L_{2}$ and $L_{t}$, respectively) are used for transforming impedance from slotline impedance to $Z_{0}$ at the sum port (port $H$ ) and at the difference port (port $E$ ), respectively. The magic- $T$ also consists of a slotline $Z_{s}$ with the length of $L_{s}$. One end of the $Z_{t}$ line is terminated with a microstrip stepped impedance openend (SIO) stub to produce a broadband virtual ground for the MS-SL transition. The SIO stub consists of the microstrip lines with the characteristic impedances of $Z_{t 1}$ and $Z_{t 2}$ and the associated electrical lengths of $\theta_{11}$ and $\theta_{l 2}$, respectively. $Z_{i 1}$ and $Z_{t 2}$ have the physical widths and lengths of $W_{t 1}$ and $W_{t 2}$, and $L_{t 1}$ and $L_{t 2}$, respectively.

Both ends of the slotline $Z_{\mathrm{s}}$ are terminated with the slotine stepped circular ring (SCR) [6] to provide broadband and lowloss MS-SL transition and to allow out-of-phase combining at MS-SL tee junction along $A-B$ in Fig. 2(a). The signals from port 1 and port 2 are combined out-of-phase at the MS-SL tee junction along $A-B$ plane and combined in-phase at the port $\mathrm{H}$ as shown in Fig. 2(a) and (b), respectively.

In the odd mode, the signals from port 1 and port 2 are outof-phase. This creates a microstrip virtual ground plane along the $y$-axis of the magic-T. The slotline SCR connected to the slotline $Z_{s l}$ also allows the MS-SL mode conversion to occurs as demonstrated by the electric-field (E-field) and current directions around the $A-B$ cross section as shown in Fig 2(a). 


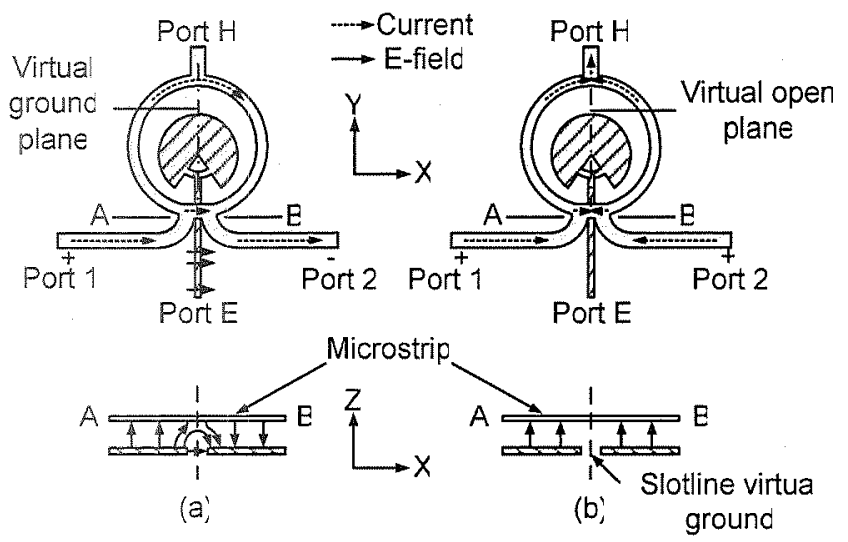

Fig. 2. (a) The odd-mode and (b) the even-mode electric field and the current flow in the magic-T.

In the even mode, the signals from port 1 and port 2 are inphase, thus creating a microstrip virtual open along the y-axis of the magic-T as shown in Fig. 2(b). E-fields in the slotline at the $A-B$ cross section are canceled thus creating a slotline virtual ground that prevents the signal flow to or from port $E$.

\section{CIRCUIT MODEL}

In order to match the impedance of all four ports of the magic- $T$, the magic- $T$ is analyzed at the center frequency in odd-mode and even-mode circuits up to the MS-SL tee junction.

In the odd mode, as shown in Fig. 3(a), the $\lambda / 4$-line $Z_{1}$ is used to transform the input characteristic impedance $Z_{0}$ at port 1 to the desired value of $Z_{\S} / 2$. The slotline SCR has no effect on the circuit at the center frequency since it is a virtual open at that frequency. Therefore, $Z_{1}$ can be derived as follows:

$$
Z_{1}=\sqrt{n_{t}{ }^{2} \frac{Z_{s}}{2} \cdot Z_{0}}
$$

where $n_{t}$ is the MS-SL transformer ratio [6]. The $\lambda / 4$-line $Z_{2}$ is used to transform the grounded-end at port $\mathrm{H}$ to a virtual open at $Z_{s}$. The practical value of $Z_{2}$ is set by the impedance matching in the even-mode analysis.

In the even mode, as shown in Fig. 3(b), the input impedance $Z_{0}$ at port 1 is transformed to the in-phase port impedance of $2 Z_{0}$. Since the line $Z_{1}$ is used to transform impedance $Z_{0}$ to $Z_{s} / 2$ in odd-mode, the line $Z_{2}$ must be used to transform the odd-mode impedance of $Z_{\mathrm{s}} / 2$ to $2 Z_{0}$. Therefore, $Z_{2}$ can be computed as follows:

$$
Z_{2}=\sqrt{2 Z_{0} \cdot n_{t}{ }^{2} \frac{Z_{s}}{2}}=\sqrt{2} Z_{1}
$$

The isolation between port 1 and port 2 and the return loss of port 1 and port 2 are derived in term of $\Gamma_{+-}$and $\Gamma_{++}$defined in Fig. 3(a) and 3(b), respectively, as follows

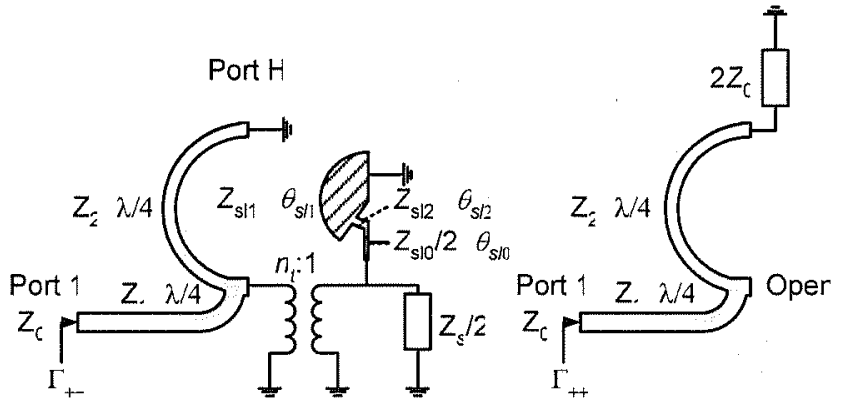

$\left(a^{\prime}\right.$

(b)

Fig. 3. (a) The odd-mode and (b) the even-mode equivalent circuit of the compact magic-T.

$$
\begin{gathered}
\text { Isolation }=-20 \log \left(\frac{\left|\Gamma_{++}-\Gamma_{+-}\right|}{2}\right) \\
\text { Return loss }=-20 \log \left(\frac{\left|\Gamma_{++}+\Gamma_{+-}\right|}{2}\right) .
\end{gathered}
$$

The magic-T is designed on a 0.25 mm-thick Duroid 6010 substrate with the dielectric constant of 10.2. The slotline is $0.1 \mathrm{~mm}$ wide, which is the minimum width allowable in this fabrication process. This corresponds to the $Z_{s}$ value of 72.8 Ohm. Given $Z_{0}=50 \mathrm{Ohm}$ and $n_{t}=1$, from (1) and (2), we obtain $Z_{1}$ and $Z_{2}$ of $42.7 \mathrm{Ohm}$ and $60.4 \mathrm{Ohm}$, respectively.

Using the circuit model in Fig 3(a) and (b), and the parameters at $10 \mathrm{GHz}$ in Table I, the frequency response of the magic-T can be determined up to the tee junction as shown in Fig. 4. This magic-T provides better broadband out-of-phase combining response than the in-phase combining response. The in-phase combining bandwidth is limited by the two impedance transformation sections in $Z_{1}$ and $Z_{2}$ used to transform $Z_{0}$ at port 1 to $2 Z_{0}$ at port $\mathrm{H}$ in even mode. Moreover, the $Z_{2}$ value needs to satisfy the odd-mode matching condition.

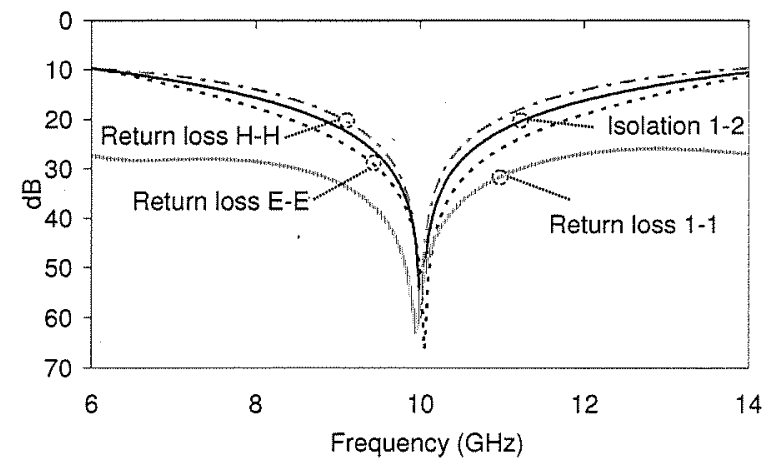

Fig. 4. The magic-T's frequency responses of the return loss and isolation using odd and even-mode circuit models. 


\section{SLOTLINE SCR TERMINATIONS IN THE PROPOSED MAGIC-T}

A SL termination is used at the MS-SL tee junction to provide a slotline virtual open and allow mode conversion in the out-of-phase combiner. It is also used in the MS-SL transition at port E. A slotline SCR termination, developed in our previous work [6] was selected for use in this magic-T due to its compact size. The slotline SCR termination minimizes the effect of parasitic in the slotline on the $Z_{2}$ lines and reduces the loss due to slotline radiation.

This slotline SCR termination can be modeled as stepped impedance transmission lines as shown in Fig. 3(a). Its equivalent circuit parameters and its physical parameters are provided in Table I and Table II, respectively. The full circuit model at $10 \mathrm{GHz}$ shows a good agreement with electromagnetic (EM) simulation result as shown in Fig. 5.

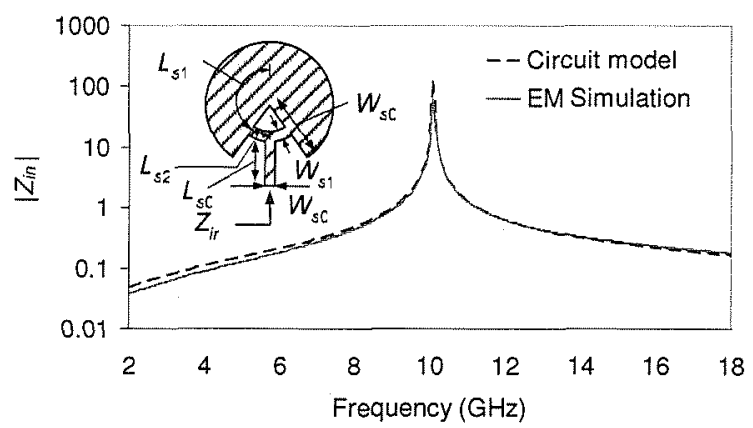

Fig. 5. The input impedance of the slotline SCR in the compact magic-T using the parameters provided in Table II.

TABLE I

THE MAGIC-T CIRCUIT DESIGN PARAMETERS AT $10 \mathrm{GHz}$

\begin{tabular}{|c|c|}
\hline $\begin{array}{c}\text { Microstrip line } \\
\text { section }\end{array}$ & Slotline section \\
\hline$Z_{1}=42.7 \Omega, Z_{2}=60.33$ & $Z_{s}=72.8 \Omega, Z_{s 0}=72.8 \Omega, Z_{s l 2}=72.8$ \\
$\Omega_{s} Z_{t 1}=40 \Omega \theta_{t 1}=23.3^{\circ}$, & $\Omega, \theta_{s 10}=13.57^{\circ}, \theta_{s / 2}=6.2^{\circ}, Z_{s / 1}$ \\
$\theta_{t 2}=46.6^{\circ}, Z_{L}=20 \Omega$ & $=163.4 \Omega, \theta_{S 11}=34.95^{\circ}, \theta_{s}=113.3^{\circ}$ \\
\hline
\end{tabular}

TABLE II

THE PHYSICAL PARAMETERS OF THE COMPACT MAGIC-T IN MILLIMETERS

\begin{tabular}{|c|r|}
\hline Microstirp line section & Slotline section \\
\hline$L_{1}=2.62, W_{1}=0.26, L_{2}=1.83$, & $L_{s}=1.92, W_{s}=0.10$ \\
$W_{2}=0.14, L_{t}=2.80, W_{t}=0.16$, & $L_{s 0}=0.58, W_{s 0}=0.10$ \\
$L_{n 1}=0.68, W_{t}=0.37, L_{2}=1.30$, & $L_{s 1}=0.23, W_{s 1}=0.10$ \\
$W_{t 2}=1.05$ & $L_{s 2}=0.91, W_{s 2}=0.71$ \\
\hline
\end{tabular}

\section{HARDWARE AND EXPERIMENTAL RESULTS}

The magic- $T$ is fabricated on a $0.25 \mathrm{~mm}$-thick Duroid 6010 substrate. The physical dimensions shown in Fig. 1 are computed based on the circuit parameters in Table I and their values are shown in Table II. The method of moments simulation is performed using Ansoft designer software [7]. Since the lines $L_{1}$ and $L_{2}$ are close to the slotline and the slotline SCR, they have less electric-field to the ground plane. Therefore $L_{1}$ and $L_{2}$ are compensated for this effect and they are slightly longer than a $\lambda / 4$ microstrip line at $10 \mathrm{GHz}$.

The photograph of the top and the bottom side of the proposed magic-T is shown in Fig 6(a) and (b), respectively. Each port is connected to a $2.4 \mathrm{~mm}$ end-launch connector. Using thru-reflect-line calibration, these connections are deembedded up to the reference plane shown in Fig. 6(a). The measurement is performed using the HP8510c network analyzer. The magic-T's in-phase and out-of-phase 3-dB power diving/combining loss are less than $0.2 \mathrm{~dB}$ and $1 \mathrm{~dB}$ from $8 \mathrm{GHz}$ to $12 \mathrm{GHz}$, respectively, as shown in Fig. 7. The return loss frequency responses in Fig. 8 and Fig. 9 are narrower than those predicted by the circuit model due to strong parasitic around the MS-SL tee junction, which requires additional transmission length $L_{1}$ and $L_{2}$ compensations. The magic- $T$ isolation response shown Fig. 10 is in good agreement with the simulation results. The magic- $T$ provides the minimum isolation of $31 \mathrm{~dB}$ in the pass band from $2 \mathrm{GHz}$ to $17 \mathrm{GHz}$.

The amplitude imbalance of the magic- $T$ is less than $0.3 \mathrm{~dB}$ from $2 \mathrm{GHz}$ to $16 \mathrm{GHz}$ as shown in Fig. 11 (a). The phase imbalance of the port $\mathrm{H}$ and port $\mathrm{E}$ are less than $\pm 1.5^{\circ}$ and $\pm 1.6^{\circ}$, respectively as shown in Fig. 11(b). The magic-T's isolation is limited by the accuracy of the measurement, nonideal finite ground plane, and fabrication misalignment.

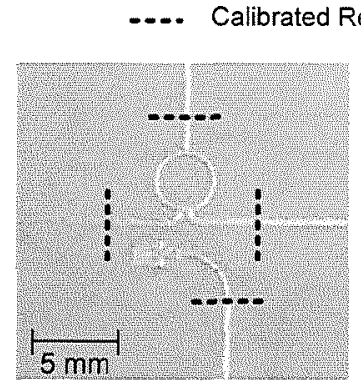

(a)

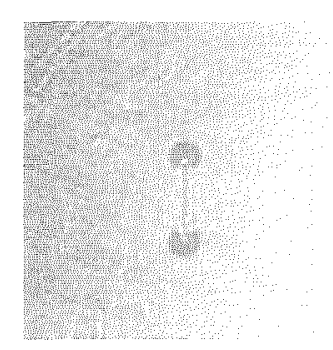

(b)
Fig. 6. The photograph of (a) the top view and (b) the bottom view of the magic-T on $0.25 \mathrm{~mm}$-thick Duroid 6010 substrate.

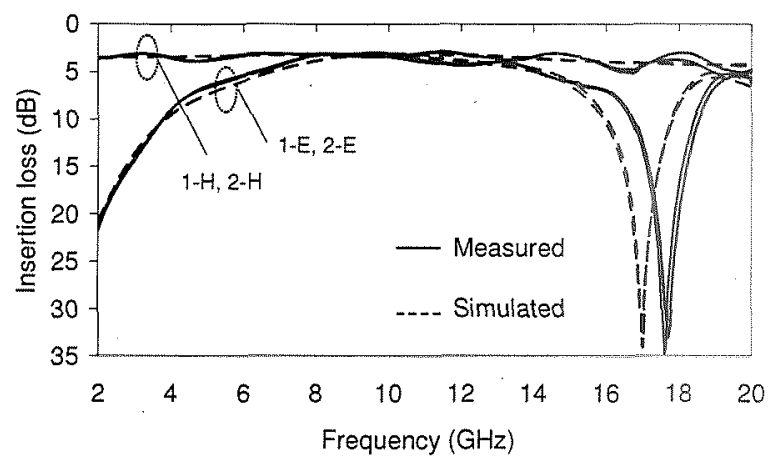

Fig. 7. The measured and simulated frequency responses of the inphase and the out-of-phase power dividing of the magic- $T$. 


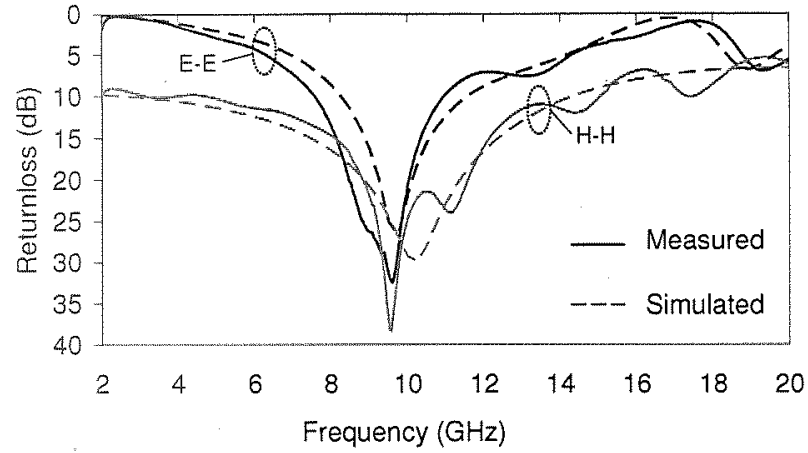

Fig. 8. The measured and simulated frequency responses of the return loss at port $\mathrm{E}$ and port $\mathrm{H}$ of the magic- $\mathrm{T}$.

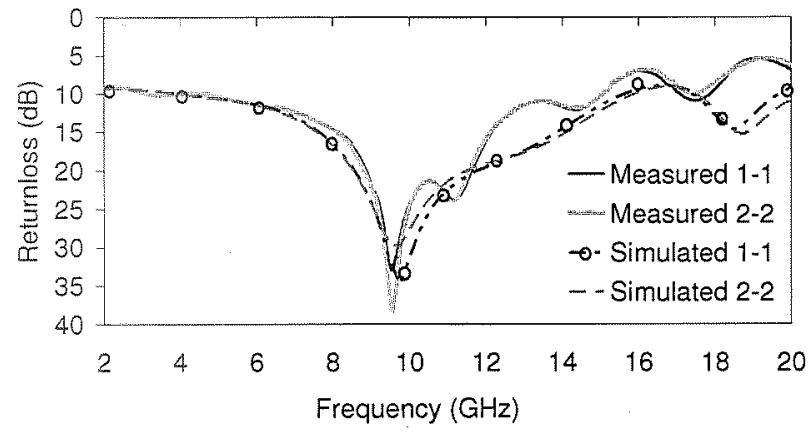

Fig. 9. The measured and simulated frequency responses of the return loss at port 1 and port 2 of the magic-T.

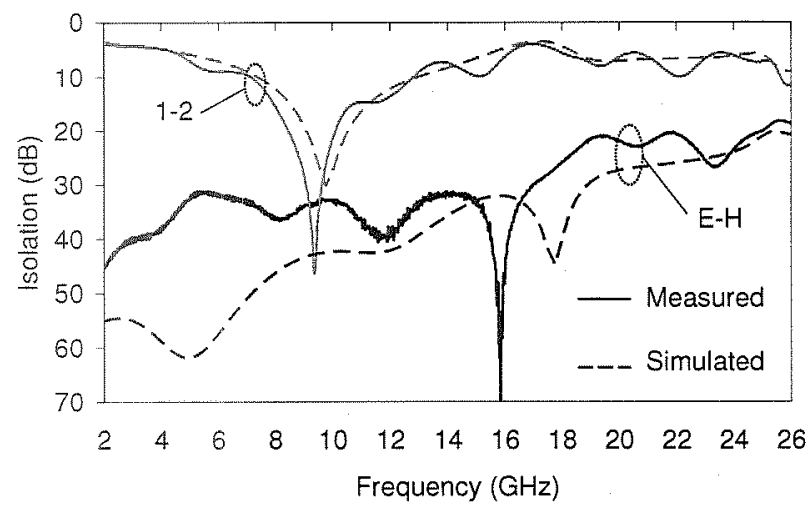

Fig: 10. The measured and simulated frequency responses of port 1 2 and port E-H isolation of the magic-T.

\section{Conclusion}

A new magic-T configuration using MS-SL transitions was developed. The effort to reduce slotline radiation in the magic$T$ design is presented for the first time. As a result, the magicT produces low-loss broadband response with little amplitude and phase imbalance. Moreover, the design is less than twothird the size of those in [2] and [3]. It is also less difficult to

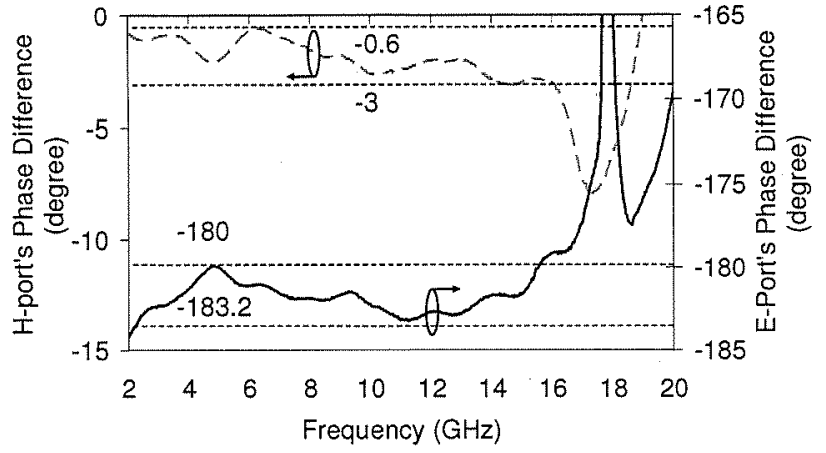

(a)

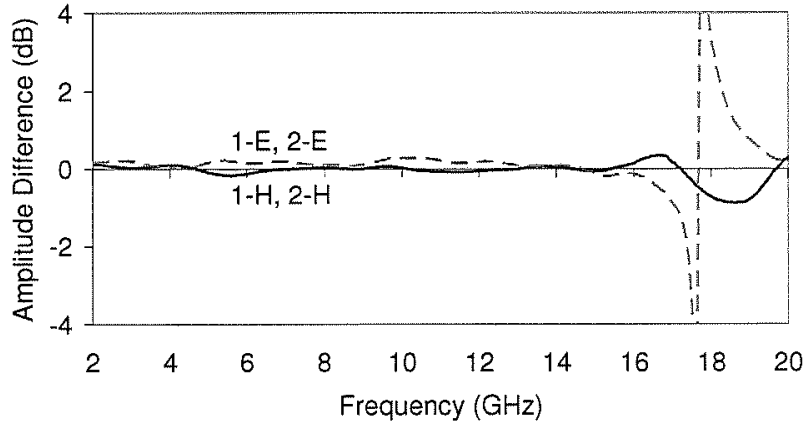

(b)

Fig. 11. The measured frequency responses of the magic-T: (a) amplitude imbalance and (b) phase imbalance.

fabricate than [1], [3]-[5] since the proposed magic-T requires no via hole or air bridges and use only two metal layers.

\section{REFERENCES}

[1] C.-H. Ho, L. Fan, and K. Chang, "New uniplanar coplanar waveguide hybrid-ring couplers and magic-T's," IEEE Trans. Microwave Theory \& Tech., vol. 42, no. 12, pp. 2440-2448, December 1994.

[2] J. P. Kim and W. S. Park, "Novel configurations of planar multilayer magic-T using microstrip-slotline transitions," IEEE Trans. Microwave Theory \& Tech., vol. 50, no. 7, pp. 16831688 , July 2002.

[3] M. Aikawa and H. Ogawa, "A new MIC magic-T using coupled slot lines," IEEE Trans. Microwave Theory \& Tech., vol. 28, no. 6, pp. 523-528, June 1980 .

[4] T. Hiraoka, T. Tokumitsu and M. Aikawa, "Very small wideband MMIC magic-T's using microstrip lines on a thin dielectric film," IEEE Trans. Microwave Theory \& Tech., vol. 37, no. 10, pp. 1569-1575, October 1989.

[5] M. Davidovitz, "A compact planar magic-T junction with aperture-coupled difference port," IEEE Microwave \& Guided Wave Lett., vol. 7, no. 8, pp. 217-218, August 1997.

[6] K. U-yen, E. J. Wollack, S. Horst, J. Papapolymerou and J. Laskar, "Slotline stepped circular rings for low-loss microstripto-slotline transitions," IEEE Microwave Wireless Comp. Lett., vol. 17, no. 2, pp. 100-102, February 2007.

[7] Ansoft Corporations, Pittsburg, PA, USA. 